\title{
Un curso de Cálculo con
}

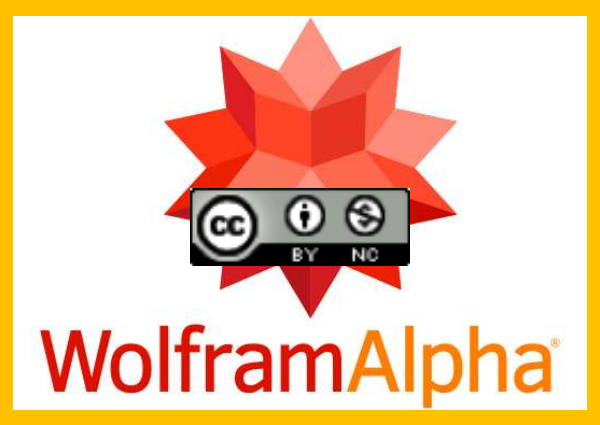

Palencia-González, F.J. y García-Llamas, M.C. Dpto. de Teoría Económica y Economía Matemática Grupo de Innovación Docente: SALT-CG

\section{UNED}

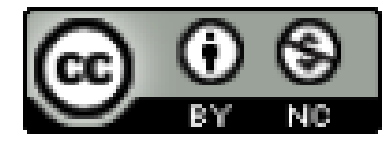




\section{Los alumnos de la UNED}

- Características generales

- Compatibilizar estudios con actividades laborales

- Cargas familiares

- Años sin estudiar

- Segundas carreras: Ingenierías vs Derecho

- Particularidades de Económicas, ADE y Turismo

- Vagos conocimientos en Matemáticas

- Muchos años sin estudiarlas

- No las han visto en los últimos cursos realizados

- Rechazo social a la materia: "ansiedad matemática" 


\section{¿Qué se estudia en estas asignaturas?}

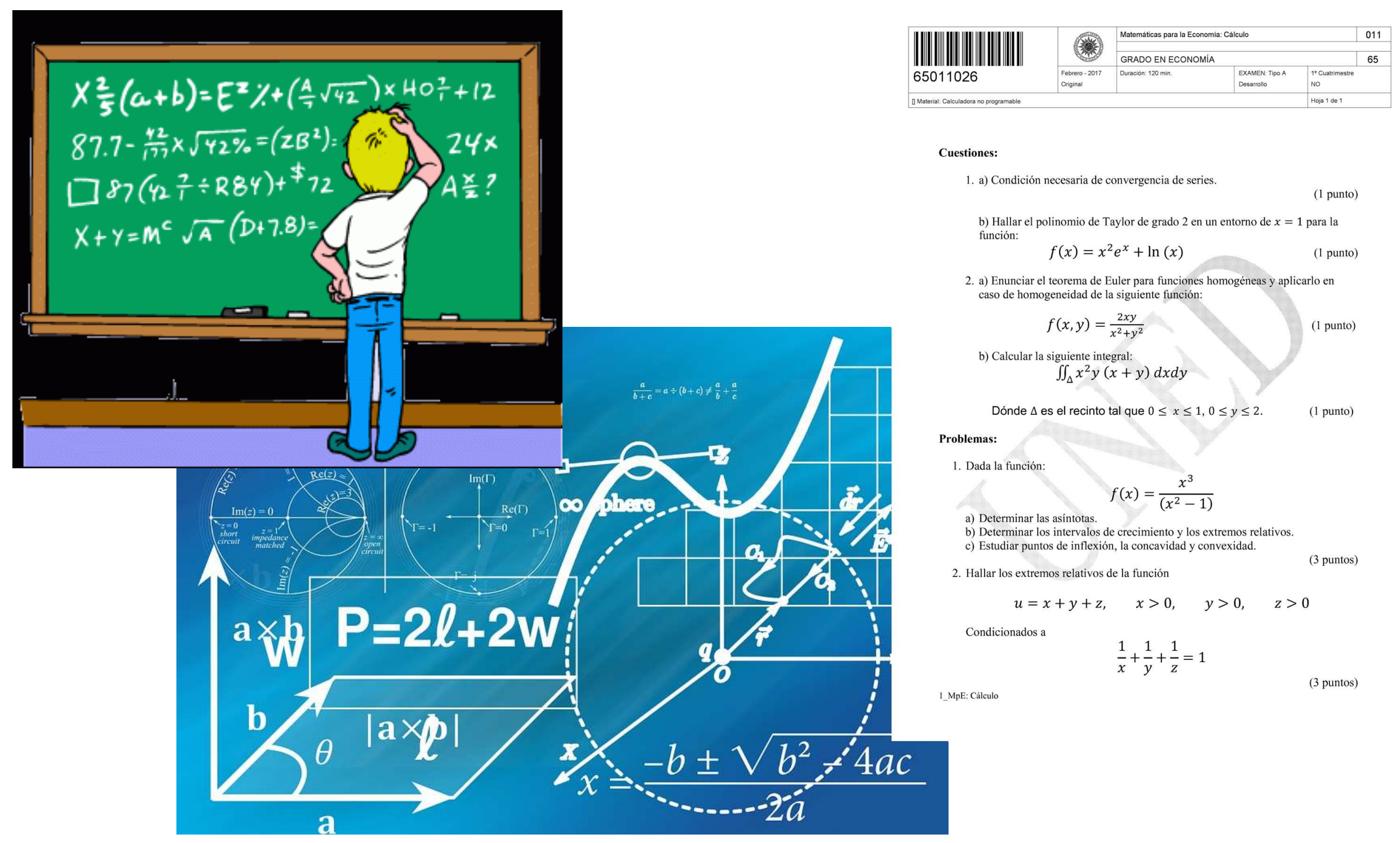




\section{Temario}

- 1.- Conceptos básicos

- 2.- Funciones

- 3.- Límites y continuidad

- 4.- Derivación

- 5.- Aplicaciones de la derivada

- 6.- La integral

- 7.- Funciones de varias variables

- 8.- Optimización de funciones, extremos condicionados e integrales múltiples

- 9.- Sucesiones y series 


\section{Soluciones}

- Explicación de la teoría:

- Tutorías presenciales

- Campus Virtual

- Libros y apuntes
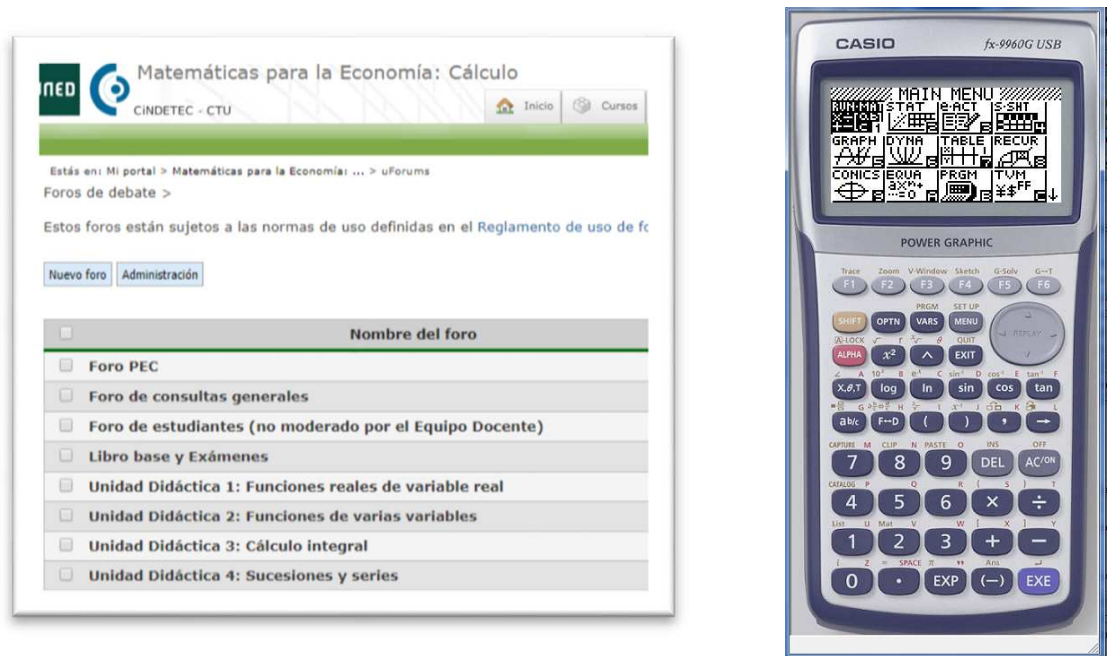

- Resolución de problemas:

- El profesor en casa

- Calculadora científica

- Un auto corrector

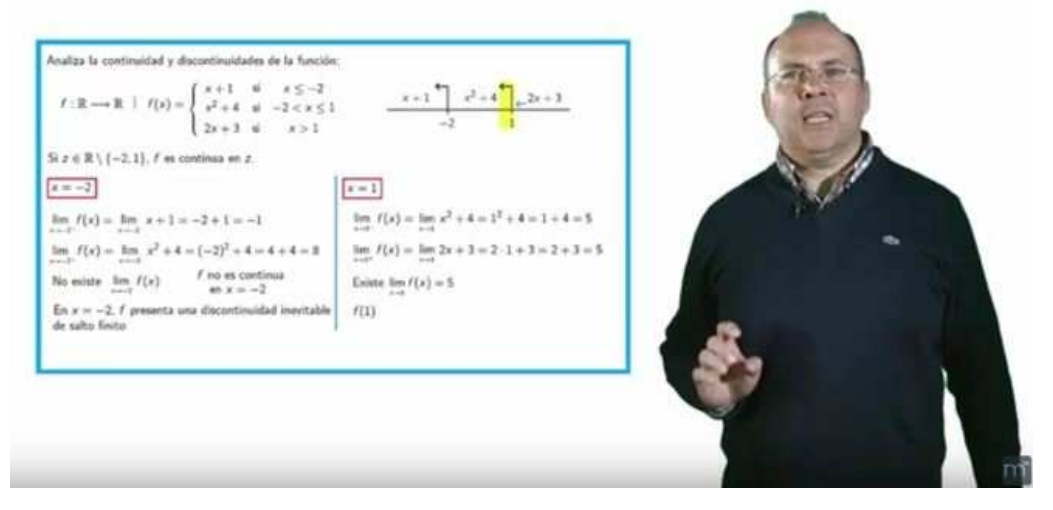




\section{Herramientas informáticas}

- Software libre
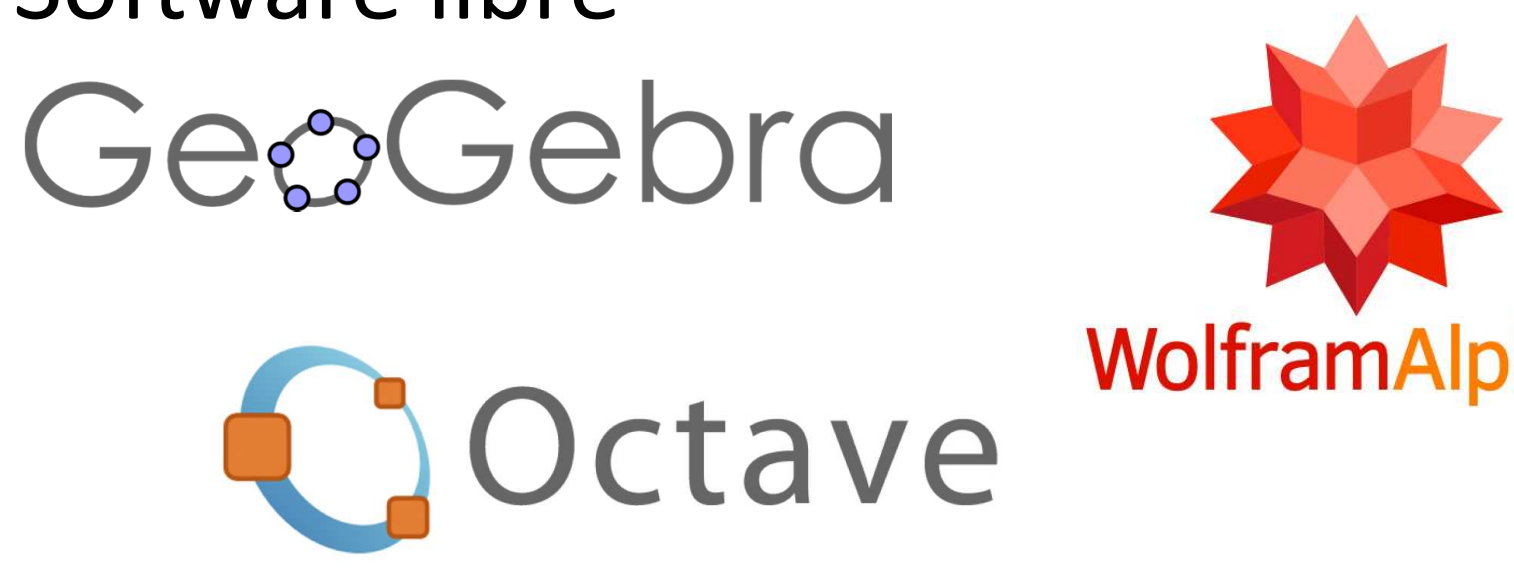

WolframAlpha

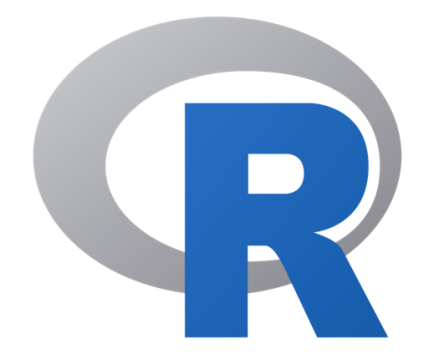

- Software comercial

Wolfram

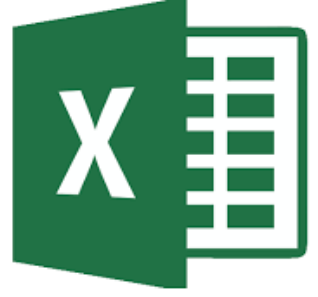

Mathematica
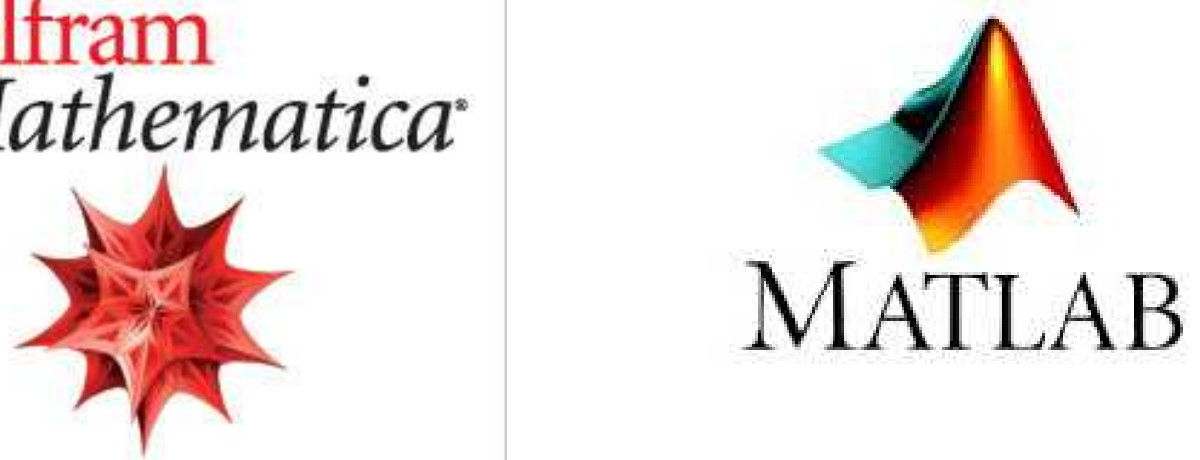


\section{Microsoft Excel}

- Serie de artículos de utilización de la hoja de cálculo para la resolución de problemas matemáticos

- Resolución de ecuaciones diferenciales

- Resolución de integrales definidas

- Resolución de ecuaciones no lineales

- Resolución de sistemas de ecuaciones lineales

- Clasificación y representación de cónicas 


\section{Microsoft Excel}

- Análisis y representación gráfica de funciones matemáticas con Excel. Premio ASEPUMA Mejor Propuesta Docente '17
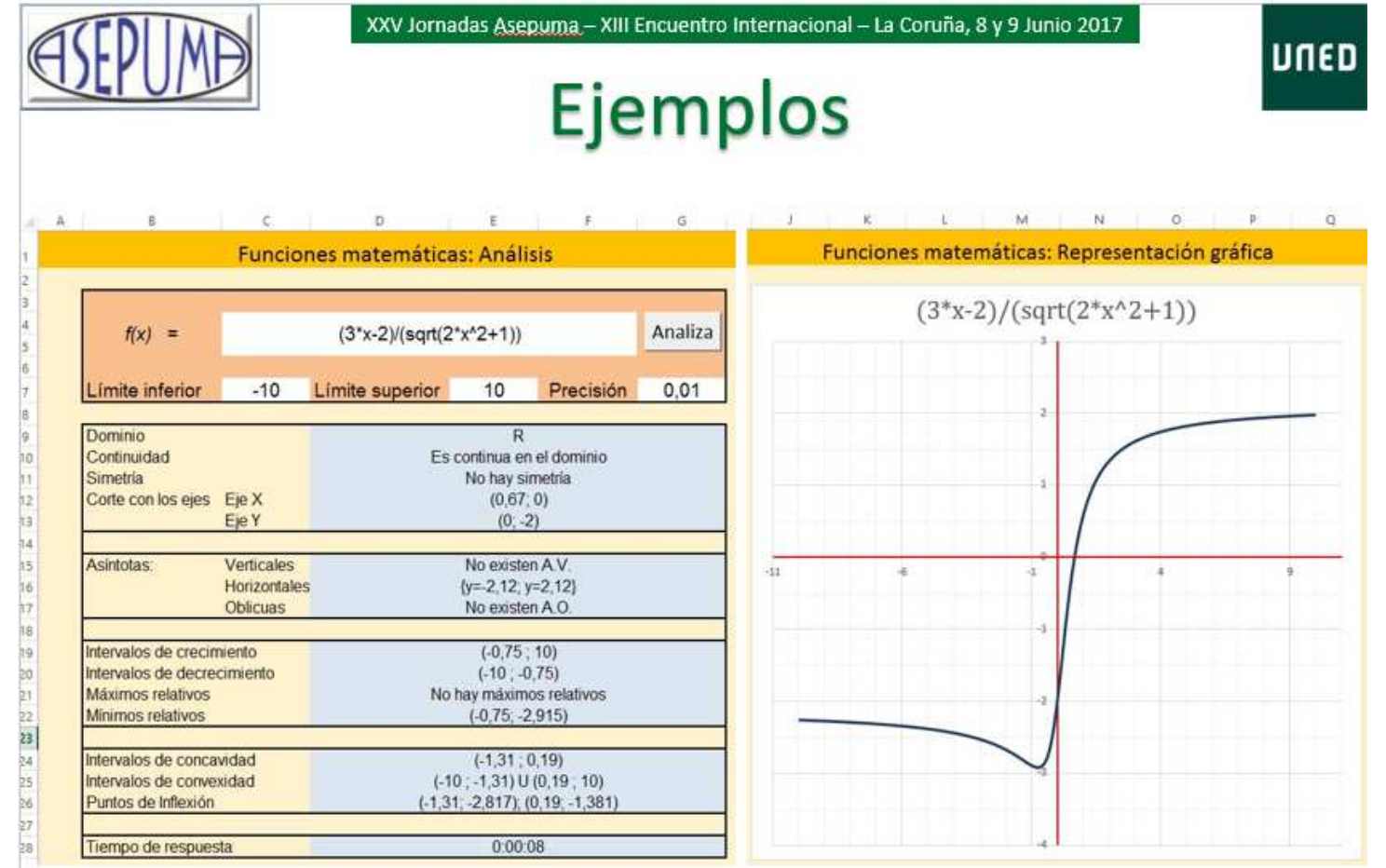


\section{Temario y Miniguías Wolfram Alpha}

1.- Conceptos básicos

2.- Funciones

3.- Límites y continuidad

4.- Derivación

5.- Aplicaciones de la derivada

6.- La integral

7.- Funciones de varias variables

8.- Optimización de funciones, extremos condicionados e integrales múltiples

9.- Sucesiones y series
Conceptos básicos

Estudio de Funciones

Cálculo de límites y continuidad

Cálculo de derivadas

Polinomio de Taylor

Estudio y representación gráfica de funciones

Cálculo de integrales

Funciones de varias variables

Determinantes funcionales y

extremos

Sucesiones y series 


\section{¿Qué es una miniguía?}

- Documento compuesto de unas 15 diapositivas

- Muy visual, acompañado de imágenes

- Sencilla y atractiva, con lenguaje directo

- Fácil de entender y de probar

- Contenidos

- Concepto a estudiar, ¿dónde encontrarlo?

- ¿Cómo escribir expresiones?

- Sintaxis de los comandos

- Ejemplos explicativos

- Hoja de ejercicios 


\section{Miniguía - Introducción}

- Conceptos a estudiar, y como llegar a ellos

$$
\text { UกED }
$$

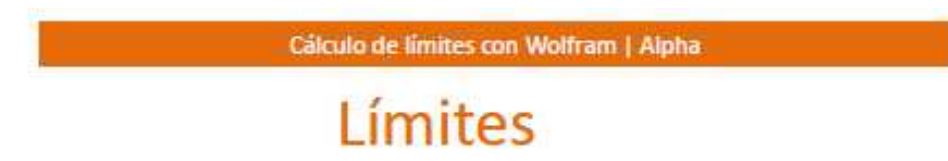

- De los diversos tipos que se pueden calcular nos interesan los límites en un punto, y los límites laterales.

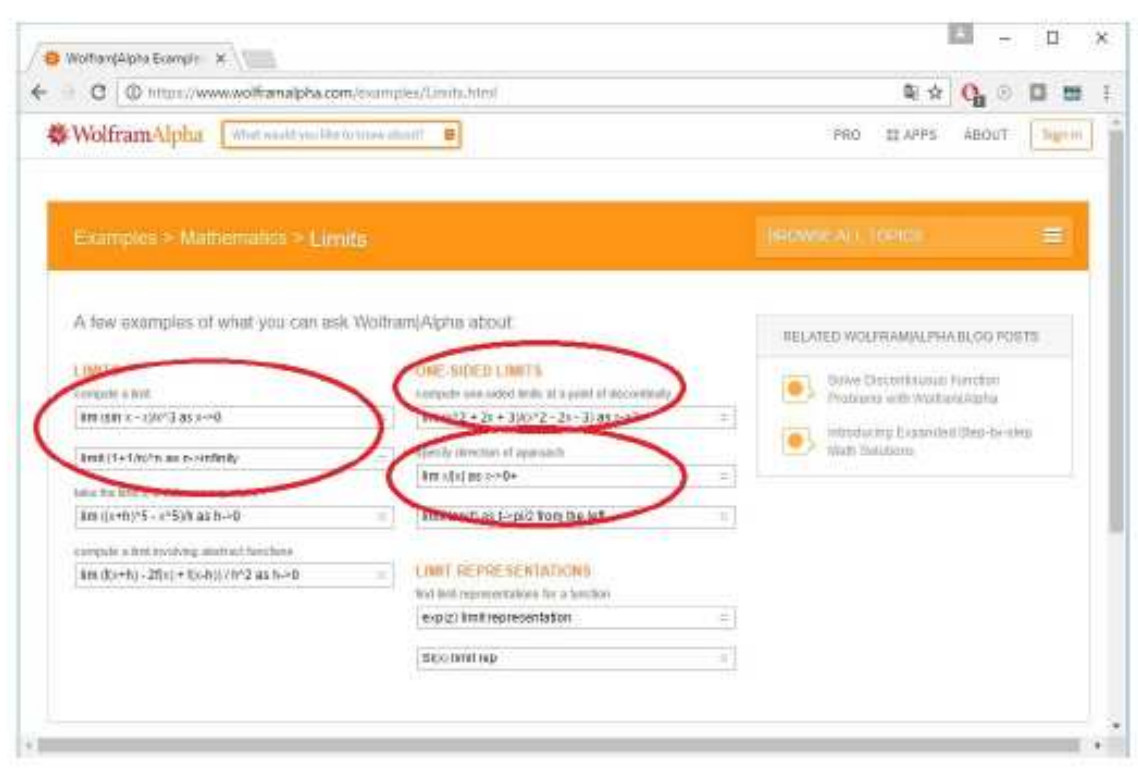




\section{Miniguía - Expresiones}

- Se explica cómo y dónde introducir las expresiones

UกED

Funciones de varias variables con Wolfram I Alpha

\section{Escribir expresiones}

- Se ha de tener cuidado con el uso de los paréntesis a la hora de introducir la función con la que queramos realizar operaciones. Los símbolos que pueden utilizarse son:
"+", suma
"*”, multiplicación
"^", potencia
"-", resta
"/", división
"sqrt", raíz cuadrada

- El valor al que tiende la variable puede ser un número o infinito ("infinity").

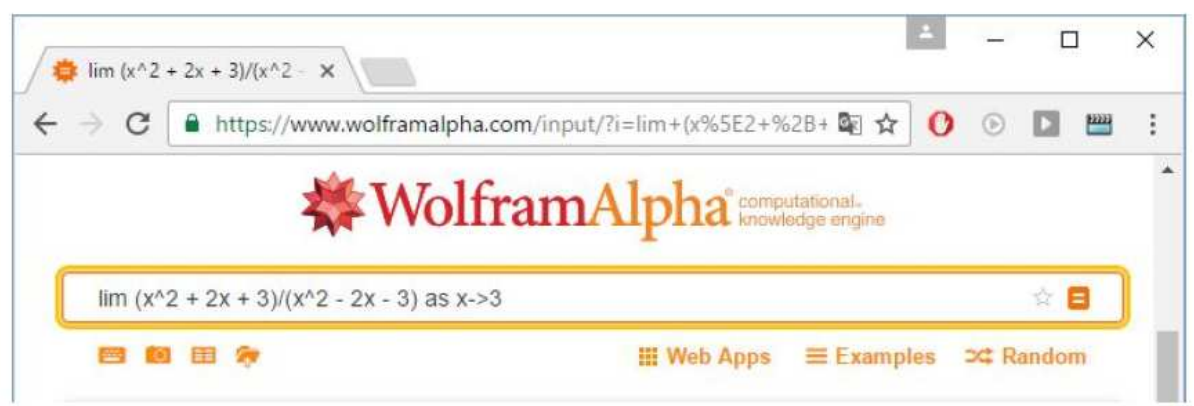




\section{Miniguía - Sintaxis}

\section{- Se introduce la sintaxis de los comandos}
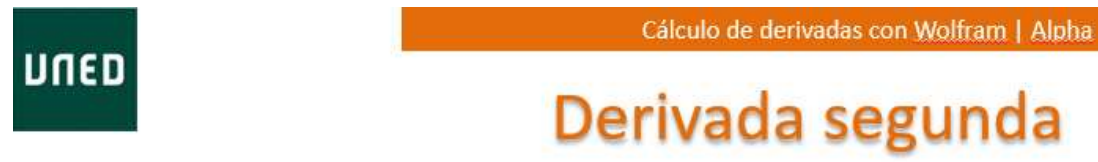

- Para calcular la segunda derivada de una función podemos hacerlo de dos formas:

- Una forma es introduciendo la variable sobre la que queremos derivar dos veces, " $d^{\wedge} 2 / d x^{\wedge} 2$ ", seguida de la función de la que queremos obtener la derivada :

$d^{\wedge} 2 / d x^{\wedge} 2$ funcion

- Otra forma es mediante las palabras reservadas "second derivative of", seguida de la función que queremos derivar dos veces:

second derivative of $\left(x^{\wedge} 4 \sin x\right)$

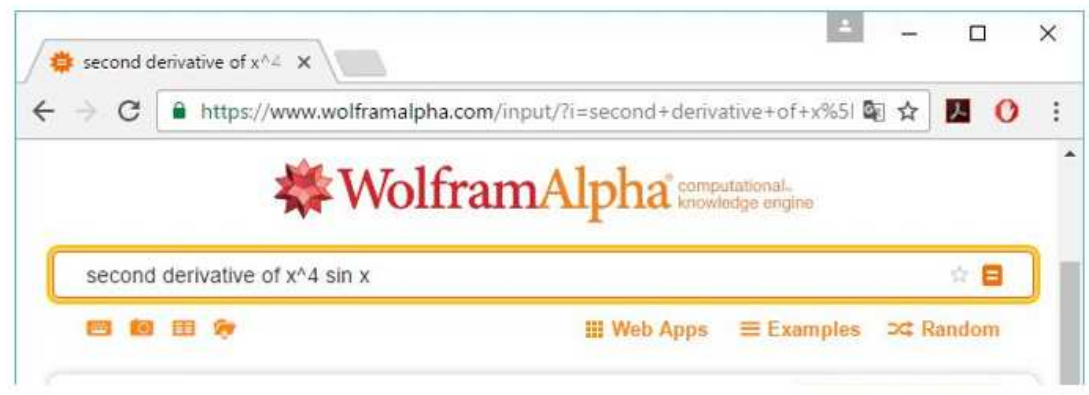




\section{Miniguía - Ejemplos}

- Se ilustra el comando con uno o varios ejemplos

\section{Jacobiano}

- Para calcular el valor del determinante Jacobiano, introducimos el determinante como hemos visto anteriormente y los valores de los elementos son las expresiones de las funciones a derivar.

$$
\operatorname{det}\left\{\left\{\frac{\partial f_{1}}{\partial x_{1}}, \frac{\partial f_{1}}{\partial x_{2}}, \ldots, \frac{\partial f_{1}}{\partial x_{n}}\right\},\left\{\frac{\partial f_{2}}{\partial x_{1}}, \frac{\partial f_{2}}{\partial x_{2}}, \ldots, \frac{\partial f_{2}}{\partial x_{n}}\right\}, \ldots,\left\{\frac{\partial f_{n}}{\partial x_{1}}, \frac{\partial f_{n}}{\partial x_{2}}, \ldots, \frac{\partial f_{n}}{\partial x_{n}}\right\}\right\}
$$

- Así por ejemplo calculamos el Jacobiano de $f_{1}(x, y)=e^{x} y^{2}, f_{2}(x, y)=e^{y} x^{2}$ :

\begin{tabular}{|c|c|c|c|}
\hline \multicolumn{2}{|c|}{$\operatorname{det}\left(\left\{\left(\left\{d / d x\left(e^{\wedge} x y^{\wedge} 2\right), d / d y\left(e^{\wedge} x y^{\wedge} 2\right)\right\},\left\{d / d x\left(\left(^{\wedge} y x^{\wedge} 2\right), d / d y\left(e^{\wedge} y x^{\wedge} 2\right)\right\}\right)\right.\right.\right.$} & \multicolumn{2}{|r|}{ 日 } \\
\hline 圈四田 & \#\#: Web Apps & $\equiv$ Examples & $x_{4}$ Random \\
\hline \multicolumn{4}{|l|}{ Input interpretation: } \\
\hline \multirow{4}{*}{$\left|\begin{array}{ll}\frac{\partial\left(x^{2} y^{2}\right)}{\partial x} & \frac{\partial\left(x^{x} y^{2}\right)}{\partial y} \\
\frac{\partial\left(x^{y} x^{2}\right)}{\partial x} & \frac{\partial\left(x^{y} x^{2}\right)}{\partial y}\end{array}\right|$} & & & \\
\hline & & & \\
\hline & & & \\
\hline & \multicolumn{3}{|c|}{$|m|$ is the determinant } \\
\hline Result: & & Steptes & yrstep solution \\
\hline
\end{tabular}




\section{Miniguía - Ejercicios}

- Se plantean varios ejercicios a realizar

UกED

Cálculo de Integrales con Wolfram | Alpha

\section{Ejemplos}

1.- Hallar la integral indefinida de las siguientes funciones
a) $f(x)=\left(x^{2}-1\right)$
b) $f(x)=\frac{1}{x-1}$
c) $f(x)=x^{2} \operatorname{sen}\left(x^{3}\right)$
d) $f(x)=e^{5 x}+\operatorname{sen}(x)$

2.- Hallar las siguientes integrales definidas
a) $f(x)=\cos ^{2} x, 0 \leq x \leq p i$
b) $f(x)=\frac{x}{\sqrt{x^{2}+9}}, 0 \leq x \leq 4$

3.- Hallar las siguientes integrales impropias
a) $f(x)=\frac{1}{1+x^{2}}, 0 \leq x \leq \infty$
b) $f(x)=\frac{e^{x}}{\ln (x)}, 1 \leq x \leq 2$

4.- Hallar las siguientes integrales múltiples

a) $f(x, y)=x^{2} y(x+y), 0 \leq x \leq 1,0 \leq y \leq 2$

b) $f(x, y)=\frac{x^{2} y(x+y)}{x^{2} y^{2}}, 1 \leq x \leq 2,2 \leq y \leq 3$ 


\section{Miniguía - Soluciones}

- Se muestran las soluciones a los ejercicios

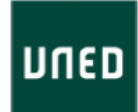

Cálculo de Integrales con Wolfram | Alpha

\section{Ejemplos}

1.- Hallar la integral indefinida de las siguientes funciones
a) $f(x)=\left(x^{2}-1\right) \operatorname{int}\left(x^{\wedge} 2-1\right) d x$
b) $f(x)=\frac{1}{x-1} \quad$ int $(1 /(x-1)) d x$
c) $f(x)=x^{2} \operatorname{sen}\left(x^{3}\right)$ int $\left(x^{\wedge} 2\right) \sin \left(x^{\wedge} 3\right) \mathrm{d} x$
d) $f(x)=e^{5 x}+\operatorname{sen}(x)$ int $\mathrm{e}^{\wedge}(5 \mathrm{x})+\sin (\mathrm{x}) \mathrm{dx}$

2.- Hallar las siguientes integrales definidas
a) $f(x)=\cos ^{2} x, 0 \leq x \leq p i$
b) $f(x)=\frac{x}{\sqrt{x^{2}+9}}, 0 \leq x \leq 4$
$\operatorname{int}(\cos (x))^{\wedge} 2, x=0$..pi
int $\mathrm{x} / \operatorname{sqrt}\left(\mathrm{x}^{\wedge} 2+9\right) \mathrm{dx}, \mathrm{x}=0 . .4$

3.- Hallar las siguientes integrales impropias
c) $f(x)=\frac{1}{1+x^{2}}, 0 \leq x \leq \infty$
d) $f(x)=\frac{e^{x}}{\ln (x)}, 1 \leq x \leq 2$
int $1 /\left(1+x^{\wedge} 2\right) d x, x=0$.. infinity
int $\mathrm{e}^{\wedge} \mathrm{x} / \ln (\mathrm{x}), \mathrm{x}=1 . .2$

4.- Hallar las siguientes integrales múltiples

a) $f(x, y)=x^{2} y(x+y), 0 \leq x \leq 1,0 \leq y \leq 2$

int $\mathrm{x}^{\wedge} 2 \mathrm{y}(\mathrm{x}+\mathrm{y}) \mathrm{dx} \mathrm{dy}, \mathrm{x}=0 . .1, \mathrm{y}=0 . .2$

b) $f(x, y)=\frac{x^{2} y(x+y)}{x^{2} y^{2}}, 1 \leq x \leq 2,2 \leq y \leq 3$

int $x^{\wedge} 2 y(x+y) /\left(x^{\wedge} 2 y^{\wedge} 2\right) d x d y, x=1 . .2, y=2 . .3$ 


\section{Miniguía - ¿Se usan?}

- Un $76 \%$ consideran útiles las miniguías (Utilidad)

- Un $67 \%$ utilizan la herramienta a la hora de preparar el examen final (Uso actual).
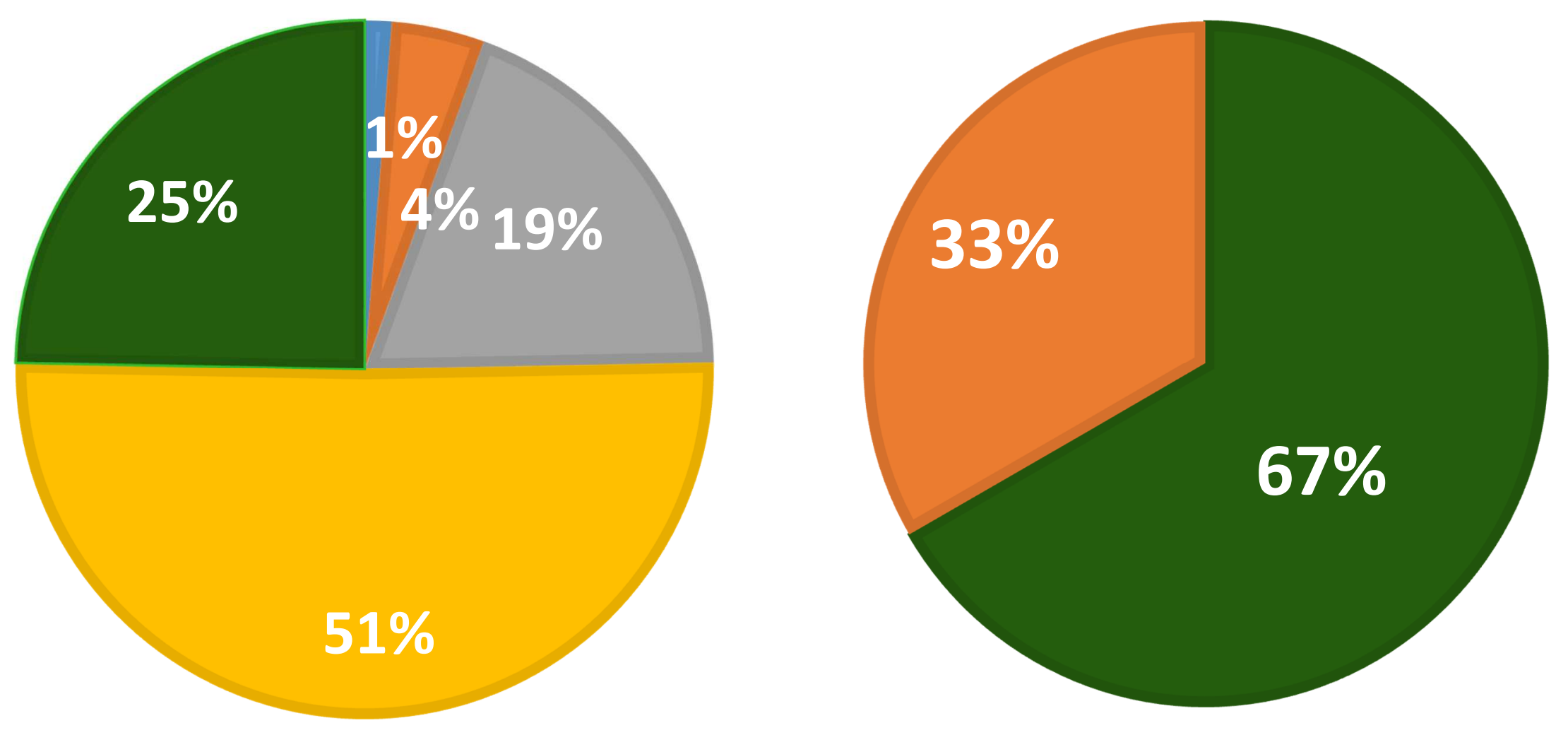


\section{Bibliografía}

- Resolución de ecuaciones diferenciales con Excel. Rect@. Mon no 4. 2013. pp 57-82.

- Resolución de integrales definidas con Excel. Anales de Asepuma. Vol 22. 2014.

- Resolución de ecuaciones no lineales con Excel. Anales de Asepuma. Vol 23. 2015.

- Resolución de sistemas de ecuaciones lineales con Excel. Anales de Asepuma. Vol 23. 2015.

- Clasificación y representación de cónicas con Excel. Anales de Asepuma. Vol 24. 2016.

- Análisis y representación gráfica de funciones matemáticas con Excel. Anales Asepuma. Vol 25. 2017.

- Uso de herramientas informáticas como apoyo a la docencia en Matemáticas. Ensayos sobre investigación en innovación y experiencias docentes en economía, empresa y turismo. 2018. Editorial UNED.

- Las TIC en la enseñanza a distancia de Matemáticas. Anales de Asepuma. Vol 26. 2018. 


\section{Recomendaciones}
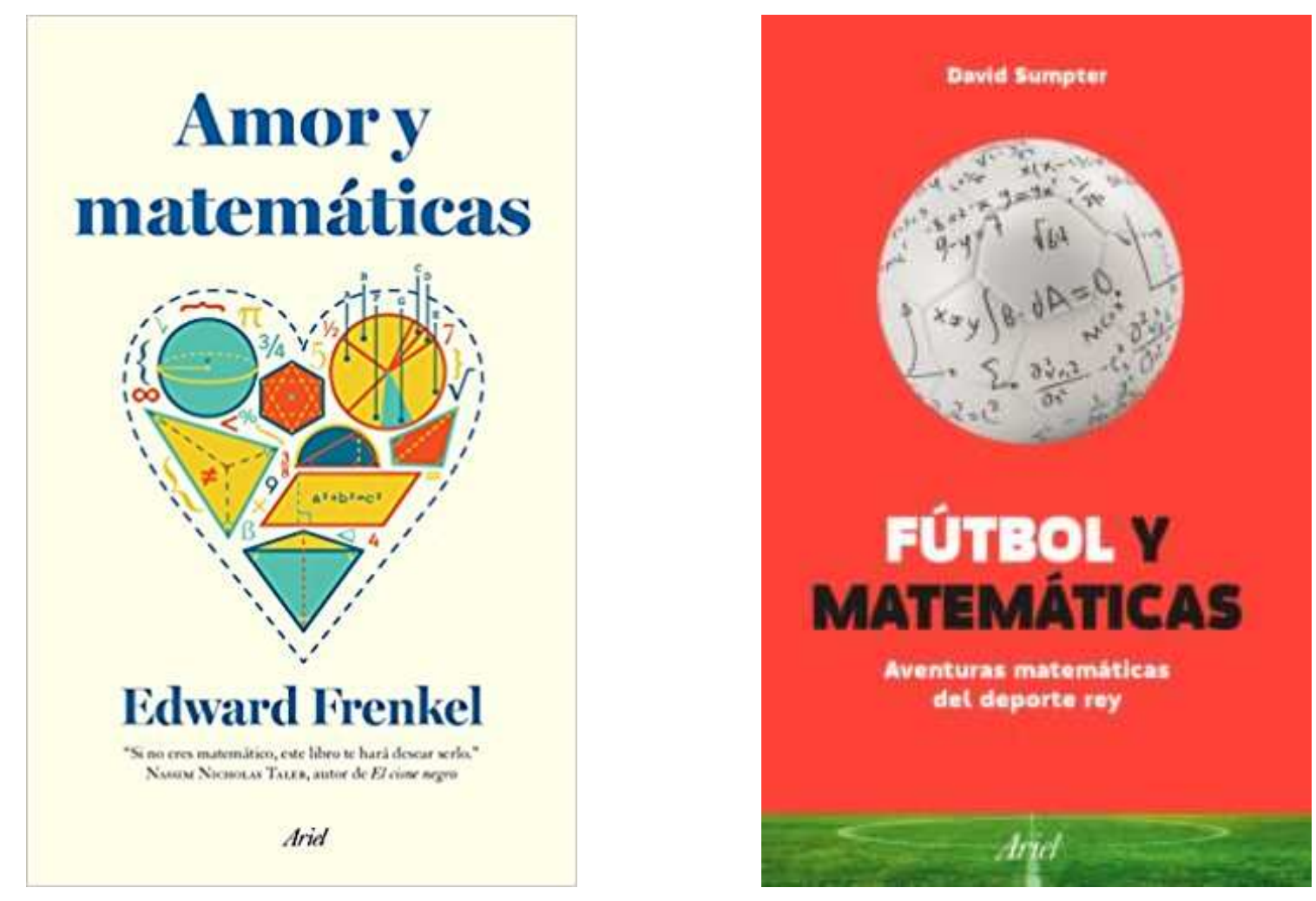


\section{Un curso de Cálculo con}

\section{Gracias por su atención}

